\section{PLAying with liposomes}

\section{By Lev Osherovich, Senior Writer}

A Danish group has developed liposomes that are prodrugs that potentially could be activated at tumor sites via a tumor-specific enzymatic reaction. ${ }^{1}$ The IP has been licensed to LiPlasome Pharma A/S, which already has an earlier version of the liposome technology for the enzymatic release of cisplatin in Phase I testing in solid tumors.

Liposomes are artificial lipid vesicles that can be used to encapsulate a therapeutic agent and protect it from degradation. In principle, liposomes can extend the activity of cancer therapeutics or deliver a payload to specific tissues and thus avoid side effects. ${ }^{2}$

In practice, however, liposomal therapeutics have shown only limited efficacy benefits over unencapsulated drugs, according to Francis Szoka Jr., professor of biopharmaceutical sciences and pharmaceutical chemistry at the University

\section{"The drug to be delivered} in its prodrug form is itself a component of the membrane. That is unique."

-Steven Deitcher, Hana Biosciences Inc. liposomes that open up only at tumor sites. ${ }^{3}$ LiPlasome's platform uses phospholipids that are specifically cleaved by sPLA $\mathrm{P}_{2}$. Andresen was formerly LiPlasome's research director and remains a scientific advisor to the company.

"It is highly interesting to design prodrugs that are specifically activated by disease-associated enzymes," he said. "This can potentially provide higher efficacy with limited side effects and damage of healthy tissue."

Andresen's next steps are to select a more suitable lipase-activated drug than capsaicin, which is not a very potent cancer therapeutic, and to

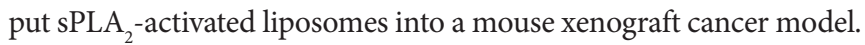

\section{Go home, liposome}

Liposome researchers cautioned that delivering the liposomal prodrug in vivo poses significant challenges.

"This is, to my knowledge, the first time that people have tried to build a prodrug into a liposome," said William Lambert, SVP of pharmaceutical development at Pacira Pharmaceuticals Inc., a spinout of drug delivery company SkyePharma plc.

Pacira markets DepoCyt(e), a liposomal formulation of cytarabine for intrathecal treatment of lymphomatous meningitis. The company's Exparel liposomal bupivacaine is in Phase III trials to treat postoperative pain.

Lambert told SciBX that Andresen's prodrug approach could potentially alter the pharmacodynamics, solubility and stability of therapeutic of California, San Francisco.

Szoka was a cofounder of Sequus Pharmaceuticals Inc., which developed Doxil doxorubicin, a liposomal cancer drug. In 1999, Sequus was acquired by Alza Corp., now a division of Johnson \& Johnson, which markets Doxil to treat a variety of cancers.

Challenges to liposomal delivery include controlling the release, localization and stability of the liposome as well as ensuring that the therapeutic payload remains active.

Now, a team at Technical University of Denmark led by Thomas Andresen, senior researcher in the Department of Micro- and Nanotechnology, has reported on the generation of liposomes incorporating a lipophilic prodrug of capsaicin and its in vitro activation via a tumorspecific enzymatic reaction.

The prodrug, consisting of capsaicin covalently bound to a phospholipid, aggregates in unilamellar (single layer) liposomes without an interior cavity. This prodrug liposome remains inactive until it is hydrolyzed by the activity of secretory phospholipase $\mathrm{A}_{2}\left(\mathrm{PLA}_{2} \mathrm{G} 2 \mathrm{~A}\right.$; $\mathrm{sPLA}_{2}$ ), an enzyme found at high concentrations in and around tumors, and the capsaicin moiety is released.

In contrast, most liposomal constructs have two lipid layers, with a drug payload in the aqueous cavity in the center of the liposome. Thus, the new technology could be useful for delivering and then activating difficult-to-dissolve lipophilic compounds.

The study, reported in Angewandte Chemie International Edition, builds on earlier efforts by Andresen's group and LiPlasome to design compounds, and he suggested that additional modifications such as a polyethylene glycol (PEG) coating likely would be needed to ensure that the liposomes indeed reach tumors.

"Regular liposomes get cleared from plasma at a fairly rapid rate because the body's reticular endothelial system recognizes them as foreign matter," he noted.

Szoka was also concerned that the in vivo behavior of liposomes may not mirror what Andresen saw in vitro. He noted that sPLA $\mathrm{PL}_{2}$-activated liposomes could build up in the liver or spleen, the natural depots for foreign particles, and could become activated in those tissues before they reach tumors, thus decreasing their efficacy and potentially causing side effects.

"Phospholipase $\mathrm{A}_{2}$ is enriched in the tumor, but it's also present elsewhere," said Szoka.

Steven Deitcher, president and CEO of Hana Biosciences Inc., noted that Andresen's lipophilic prodrug strategy contrasted with the more standard approach of packing water-soluble drugs into the aqueous space within the liposome.

"The drug to be delivered in its prodrug form is itself a component of the membrane," said Deitcher. "That is unique-there is no payload in the core of the liposome."

Hana's Marqibo liposomal vincristine is in Phase III trials for acute lymphoblastic leukemia (ALL). Marqibo was developed by Tekmira Pharmaceuticals Corp., itself a spinout of Inex Pharmaceuticals 
Corp., and licensed to Hana in 2006 after the formulation received a nonapprovable letter from the FDA in 2005.

Deitcher also wondered whether other compounds would be as amenable to spontaneous aggregation in unilamellar vesicles as capsaicin. "Spontaneous aggregation in unilamellar vesicles could be unique to capsaicin," he said.

Andresen said marketed chemotherapeutics that would be chemically compatible with the approach include cytarabine, GlaxoSmithKline plc's Hycamtin topotecan and Pfizer Inc.'s Camptosar irinotecan.

Indeed, LiPlasome is using an earlier version of Andresen's sPLA ${ }_{2}^{-}$ activatable liposome technology to encapsulate chemotherapeutics in liposomes that release their cargo upon hydrolyzation of their phospholipid membrane by $\mathrm{sPLA}_{2}$. The company's LiPlaCis liposomal formulation of cisplatin is in a Phase I trial in solid tumors.

Osherovich, L. SciBX 2(6); doi:10.1038/scibx.2009.215

Published online Feb. 12, 2009

\section{REFERENCES}

1. Linderoth, L. et al. Angew. Chem. Int. Ed. Engl.; published online Feb. 5, 2009; doi:10.1002/anie.200805241

Contact: Thomas L. Andresen, Technical University of Denmark, Roskilde, Denmark

e-mail: thomas.andresen@nanotech.dtu.dk

2. Guo, X. \& Szoka, F.C. Jr. Acc. Chem. Res. 36, 335-341 (2003)

3. Andresen, T.L. et al. J. Med. Chem. 47, 1694-1703 (2004)

COMPANIES AND INSTITUTIONS MENTIONED

GlaxoSmithKline plc (LSE:GSK; NYSE:GSK), London, U.K.

Hana Biosciences Inc. (NASDAQ:HNAB), South San Francisco, Calif. Inex Pharmaceuticals Corp., Burnaby, British Columbia, Canada Johnson \& Johnson (NYSE:JNJ), New Brunswick, N.J.

LiPlasome Pharma A/S, Lyngby, Denmark

Pacira Pharmaceuticals Inc., Parsippany, N.J.

Pfizer Inc. (NYSE:PFE), New York, N.Y.

SkyePharma plc (LSE:SKP), London, U.K.

Technical University of Denmark, Lyngby, Denmark

Tekmira Pharmaceuticals Corp. (TSX:TKM), Burnaby,

British Columbia, Canada

University of California, San Francisco, Calif. 